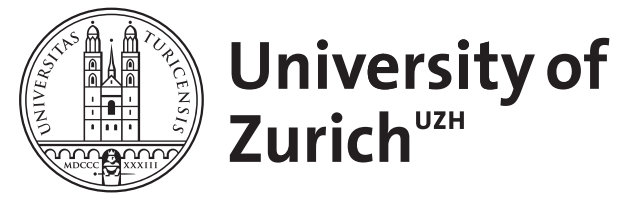
Archive

University of Zurich

University Library

Strickhofstrasse 39

CH-8057 Zurich

www.zora.uzh.ch

Year: 2011

\title{
Le carré du foncteur de dualité est monoïdal
}

Ayoub, J

\begin{abstract}
Soit (c, ) une catégorie monoïdale fermée (mais non nécessairement symétrique). Soient $\mathrm{R}$ un objet dualisable de c, et et les foncteurs de dualité associés. Dans cette note, on montre que et sont des foncteurs monoïdaux.
\end{abstract}

DOI: https://doi.org/10.1080/00927871003591868

Other titles: The square of the duality functor is monoidal

Posted at the Zurich Open Repository and Archive, University of Zurich

ZORA URL: https://doi.org/10.5167/uzh-50939

Journal Article

Accepted Version

Originally published at:

Ayoub, J (2011). Le carré du foncteur de dualité est monoïdal. Communications in Algebra, 39(5):15281535.

DOI: https://doi.org/10.1080/00927871003591868 


\title{
LE CARRÉ DU FONCTEUR DE DUALITÉ EST MONOÏDAL
}

\author{
par
}

Joseph Ayoub

\begin{abstract}
Résumé. - Soit $(\mathcal{C}, \otimes)$ une catégorie monoïdale fermée (mais non nécessairement symétrique). Soient $R$ un objet dualisable de $\mathcal{C}$ et $\mathrm{D}_{g}=\underline{\operatorname{Hom}}_{g}(-, R)$ et $\mathrm{D}_{d}=\underline{\operatorname{Hom}}_{d}(-, R)$ les foncteurs de dualité associés. Dans cette note, on montre que $\mathrm{D}_{g}^{2}$ et $\mathrm{D}_{d}^{2}$ sont des foncteurs monoïdaux.
\end{abstract}

Table des matières

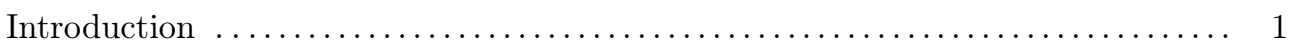

1. Les structures monoïdales $\hat{\otimes}_{g}$ et $\hat{\otimes}_{d}$ et l'énoncé du résultat principal $\ldots \ldots \ldots 2$

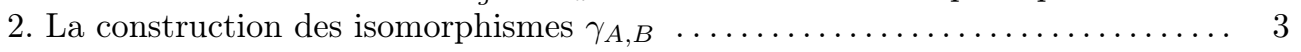

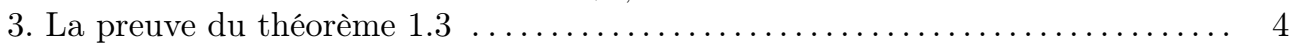

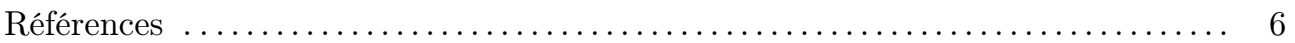

\section{Introduction}

Une structure monoïdale sur une catégorie $\mathcal{C}$ est un couple $(-\otimes-, \sigma)$ constitué d'un bifoncteur $-\otimes-$ et d'une transformation naturelle inversible $\sigma:(-\otimes-) \otimes-\simeq-\otimes(-\otimes-)$ telle que le diagramme du pentagone commute (voir [2]). Le triplet $(\mathcal{C}, \otimes, \sigma)$ est appelé une catégorie monoïdale. Dans la suite, nous omettrons la mention des isomorphismes d'associativité $\sigma$. Nous sommes surtout intéressés par le cas où la structure monoïdale sur $\mathcal{C}$ n'est pas symétrique (et plus précisément, n'est pas munie d'isomorphismes de commutativité).

Soit $(\mathcal{C}, \otimes)$ une catégorie monoïdale fermée à droite et à gauche (au sens de [1, 2.1.119]). Pour un objet $A$ de $\mathcal{C}$, on notera $\underline{\operatorname{Hom}}_{g}(A,-)$ et $\underline{\operatorname{Hom}}_{d}(A,-)$ les adjoints à droite respectifs des foncteurs $A \otimes-$ et $-\otimes A$. Pour tout objet $R$ de $\mathcal{C}$ on dispose de deux transformations naturelles en $A$ (voir [1, Lem. 2.1.133]) :

$$
A \longrightarrow \underline{\operatorname{Hom}}_{g}\left(\underline{\operatorname{Hom}}_{d}(A, R), R\right) \quad \text { et } \quad A \longrightarrow \underline{\operatorname{Hom}}_{d}\left(\underline{\operatorname{Hom}}_{g}(A, R), R\right) .
$$

L'objet $R$ est dit dualisant à gauche (resp. à droite) si le premier (resp. le second) morphisme est inversible pour tout $A \in \mathrm{Ob}(\mathcal{C})$. Il est dualisant s'il l'est à gauche et à droite.

Dans la suite on suppose donné un objet dualisant $R$ et on notera $\mathrm{D}_{g}(-)=\underline{\operatorname{Hom}}_{g}(-, R)$ et $\mathrm{D}_{d}(-)=\underline{\operatorname{Hom}}_{d}(-, R)$ les foncteurs de dualité. Les deux foncteurs (contravariants) $\mathrm{D}_{g}$ et $\mathrm{D}_{d}$ sont donc des équivalences inverses l'une de l'autre. Il est facile de se convaincre que ces équivalences ne sont pas monoïdales en général. Lors d'une communication privée avec l'auteur, Drinfel'd a formulé l'espoir que les foncteurs $\Phi_{g}=\mathrm{D}_{g} \circ \mathrm{D}_{g}$ et $\Phi_{d}=\mathrm{D}_{d} \circ \mathrm{D}_{d}$, bien que non nécessairement isomorphes au foncteur identité, seraient monoïdaux d'une manière naturelle. Il a construit même des isomorphismes $\Phi_{g}(A \otimes B) \simeq \Phi_{g}(A) \otimes \Phi_{g}(B)$ et $\Phi_{d}(A \otimes B) \simeq \Phi_{d}(A) \otimes \Phi_{d}(B)$. Le but de cette note est de vérifier que les isomorphismes de Drinfeld sont compatibles aux isomorphismes d'associativité, prouvant ainsi que les foncteurs $\Phi_{g}$ et $\Phi_{d}$ sont monoïdaux. 
Expliquons brièvement la construction de Drinfeld. Pour tout objets $A, B \in \mathrm{Ob}(\mathcal{C})$ on dispose d'isomorphismes canoniques

Il vient que

$$
\begin{aligned}
& \operatorname{hom}_{\mathcal{C}}(A \otimes B, R) \simeq \operatorname{hom}_{\mathcal{C}}(B, \underline{\operatorname{Hom}}(B, R))=\operatorname{hom}_{\mathcal{C}}\left(B, \mathrm{D}_{g}(A)\right) \\
& \operatorname{hom}_{\mathcal{C}}(A \otimes B, R) \simeq \operatorname{hom}_{\mathcal{C}}(A, \underline{\operatorname{Hom}}(A, R))=\operatorname{hom}_{\mathcal{C}}\left(A, \mathrm{D}_{d}(B)\right)
\end{aligned}
$$

$$
\begin{gathered}
\operatorname{hom}_{\mathcal{C}}(A \otimes B, R) \simeq \operatorname{hom}_{\mathcal{C}}\left(B, \mathrm{D}_{g}(A)\right) \simeq \operatorname{hom}_{\mathcal{C}}\left(B, \mathrm{D}_{d} \Phi_{g}(A)\right) \\
\simeq \operatorname{hom}_{\mathcal{C}}\left(\Phi_{g}(A), \mathrm{D}_{g}(B)\right) \simeq \operatorname{hom}_{\mathcal{C}}\left(B \otimes \Phi_{g}(A), R\right) .
\end{gathered}
$$

Étant donné un troisième objet $C$, on considère les deux suites d'isomorphismes ci-dessous

$$
\begin{gathered}
\operatorname{hom}_{\mathcal{C}}(A \otimes(B \otimes C), R) \simeq \operatorname{hom}_{\mathcal{C}}\left((B \otimes C) \otimes \Phi_{g}(A), R\right) \simeq \operatorname{hom}_{\mathcal{C}}\left(B \otimes\left(C \otimes \Phi_{g}(A)\right), R\right) \\
\simeq \operatorname{hom}_{\mathcal{C}}\left(\left(C \otimes \Phi_{g}(A)\right) \otimes \Phi_{g}(B), R\right) \simeq \operatorname{hom}_{\mathcal{C}}\left(C \otimes\left(\Phi_{g}(A) \otimes \Phi_{g}(B)\right), R\right)
\end{gathered}
$$

et

$$
\operatorname{hom}_{\mathcal{C}}(A \otimes(B \otimes C), R) \simeq \operatorname{hom}_{\mathcal{C}}((A \otimes B) \otimes C, R) \simeq \operatorname{hom}_{\mathcal{C}}\left(C \otimes \Phi_{g}(A \otimes B), R\right) .
$$

On déduit alors un isomorphisme $\operatorname{hom}_{\mathcal{C}}\left(\Phi_{g}(A) \otimes \Phi_{g}(B), \mathrm{D}_{g}(C)\right) \simeq \operatorname{hom}_{\mathcal{C}}\left(\Phi_{g}(A \otimes B), \mathrm{D}_{g}(C)\right)$. Étant donné que $\mathrm{D}_{g}$ est une équivalence de catégories, le lemme de Yoneda fournit un isomorphisme $\Phi_{g}(A) \otimes \Phi_{g}(B) \simeq \Phi_{g}(A \otimes B)$. C'est l'isomorphisme de Drinfel'd.

\section{Les structures monoïdales $\hat{\otimes}_{g}$ et $\hat{\otimes}_{d}$ et l'énoncé du résultat principal}

Notons le lemme suivant dont la démonstration est laissée aux lecteurs :

LEMME 1.1 - Étant données une catégorie monoïdale $(\mathcal{C}, \otimes)$ et une équivalence de catégories $E: \mathcal{C} \longrightarrow \mathcal{C}^{\prime}$, il existe à un unique isomorphisme binaturel près une structure monoïdale $-\otimes^{\prime}-:$ $\mathcal{C}^{\prime} \times \mathcal{C}^{\prime} \longrightarrow \mathcal{C}^{\prime}$ faisant de $E$ un foncteur monö̈dal. De plus si $E^{-1}$ est un quasi-inverse à $E$ on peut prendre $A^{\prime} \otimes^{\prime} B^{\prime}=E\left(E^{-1}\left(A^{\prime}\right) \otimes E^{-1}\left(B^{\prime}\right)\right)$ pour $\left(A^{\prime}, B^{\prime}\right) \in \mathrm{Ob}\left(\mathcal{C}^{\prime}\right)^{2}$.

On applique le lemme précédant aux équivalences $\mathrm{D}_{g}$ et $\mathrm{D}_{d}$ :

DEFINITION 1.2 - On définit deux structures monö̈dales $-\hat{\otimes}_{g}-$ et $-\hat{\otimes}_{d}-$ sur $\mathcal{C}$ en posant

$$
A \hat{\otimes}_{g} B=\mathrm{D}_{g}\left(\mathrm{D}_{d}(A) \otimes \mathrm{D}_{d}(B)\right) \quad \text { et } \quad A \hat{\otimes}_{d} B=\mathrm{D}_{d}\left(\mathrm{D}_{g}(A) \otimes \mathrm{D}_{g}(B)\right)
$$

$\operatorname{pour}(A, B) \in \mathrm{Ob}(\mathcal{C})^{2}$.

On a ainsi deux foncteurs monoïdaux $\mathrm{D}_{g}:(\mathcal{C}, \otimes) \longrightarrow\left(\mathcal{C}, \hat{\otimes}_{g}\right)$ et $\mathrm{D}_{d}:(\mathcal{C}, \otimes) \longrightarrow\left(\mathcal{C}, \hat{\otimes}_{d}\right)$. En passant aux inverses, on a également deux autres foncteurs monoïdaux $\mathrm{D}_{g}:\left(\mathcal{C}, \hat{\otimes}_{d}\right) \longrightarrow(\mathcal{C}, \otimes)$ et $\mathrm{D}_{d}:\left(\mathcal{C}, \hat{\otimes}_{g}\right) \longrightarrow(\mathcal{C}, \otimes)$. Le résultat principal de cette note est le suivant :

Theoreme $1.3-$ Il existe des isomorphismes $\gamma_{A, B}: A \hat{\otimes}_{d} B \stackrel{\sim}{\longrightarrow} A \hat{\otimes}_{g} B$ (voir la définition 2.2) binaturels en $(A, B) \in \mathrm{Ob}(\mathcal{C})^{2}$ et induisants un isomorphisme de structures monö̈dales.

En d'autres termes, le foncteur $\operatorname{id}_{\mathcal{C}}$ muni des isomorphismes $\gamma_{A, B}$ définit un isomorphisme de catégories monoïdales $(\mathrm{id}, \gamma):\left(\mathcal{C}, \hat{\otimes}_{g}\right) \simeq\left(\mathcal{C}, \hat{\otimes}_{d}\right)$. Comme corollaire du théorème 1.3 , on obtient une structure de foncteur monoïdal sur $\Phi_{g}$ en prenant la composition des foncteurs monoïdaux

$$
(\mathcal{C}, \otimes) \stackrel{\mathrm{D}_{g}}{\longrightarrow}\left(\mathcal{C}, \hat{\otimes}_{g}\right) \stackrel{(\mathrm{id}, \gamma)}{\longrightarrow}\left(\mathcal{C}, \hat{\otimes}_{d}\right) \stackrel{\mathrm{D}_{g}}{\longrightarrow}(\mathcal{C}, \otimes)
$$

On laissera aux lecteurs le soin de vérifier, en utilisant la définition 2.2, que l'accouplement $\Phi_{g}(-) \otimes \Phi_{g}(-) \simeq \Phi_{g}(-\otimes-)$ ainsi obtenu coïncide avec l'isomorphisme de Drinfel'd décrit dans l'introduction. 


\section{La construction des isomorphismes $\gamma_{A, B}$}

On dispose d'un isomorphisme naturel

$$
c_{g}: \underline{\operatorname{Hom}}_{g}\left(A, \underline{\operatorname{Hom}}_{d}(B,-)\right) \simeq \underline{\operatorname{Hom}}_{d}\left(B, \underline{\operatorname{Hom}}_{g}(A,-)\right)
$$

obtenu par adjonction de l'isomorphisme d'associativité $A \otimes(-\otimes B) \simeq(A \otimes-) \otimes B$. On notera par $c_{d}$ l'inverse de $c_{g}$. On a également des isomorphismes naturels

$a_{g}: \underline{\operatorname{Hom}}_{g}(A \otimes B,-) \simeq \underline{\operatorname{Hom}}_{g}\left(B, \underline{\operatorname{Hom}}_{g}(A,-)\right) \quad$ et $\quad a_{d}: \underline{\operatorname{Hom}}_{d}(A \otimes B,-) \simeq \underline{\operatorname{Hom}}_{d}\left(A, \underline{\operatorname{Hom}}_{d}(B,-)\right)$ obtenus par adjonction des isomorphismes d'associativité $(A \otimes B) \otimes-\simeq A \otimes(B \otimes-)$ et $-\otimes(A \otimes B) \simeq$ $(-\otimes A) \otimes B$. Nous aurons besoin du résultat suivant :

\section{LEMME 2.1 - Le diagramme}

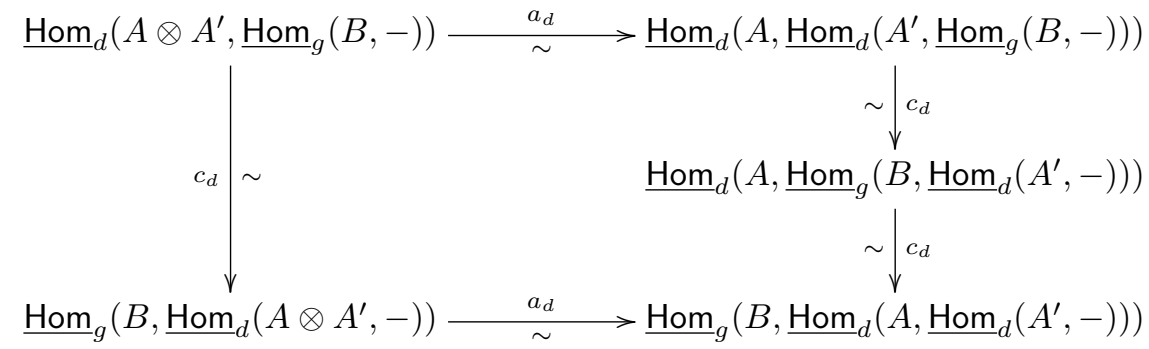

est commutatif pour $\left(A, A^{\prime}, B\right) \in \mathrm{Ob}(\mathcal{C})^{3}$.

Demonstration En passant aux adjoints à gauche, on obtient le diagramme

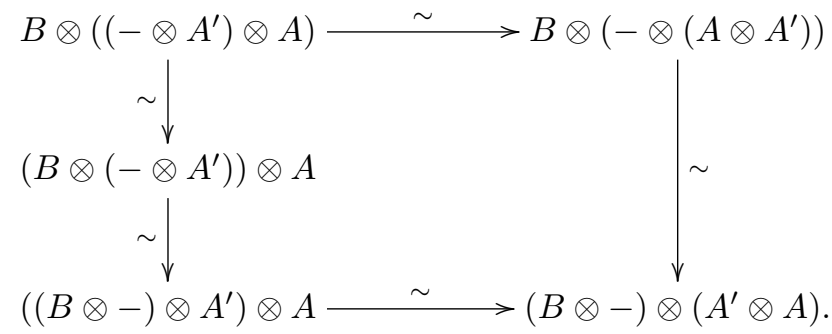

La commutativité de ce diagramme n'est autre que le bien connu axiome du pentagone.

Definition 2.2 - Soient $A$ et $B$ deux objets de $\mathcal{C}$.

1-On définit $\beta_{A, B}: \underline{\operatorname{Hom}}_{d}\left(\mathrm{D}_{g}(A), B\right) \stackrel{\sim}{\longrightarrow} \underline{\operatorname{Hom}}_{g}\left(\mathrm{D}_{d}(B), A\right)$ comme étant l'unique morphisme rendant commutatif le diagramme ci-dessous

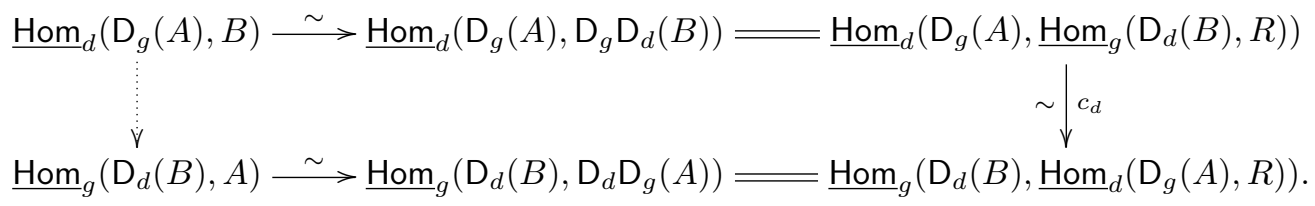

2- On définit $\gamma_{A, B}: A \hat{\otimes}_{d} B \stackrel{\sim}{\longrightarrow} A \hat{\otimes}_{g} B$ comme étant l'unique morphisme rendant commutatif le diagramme ci-dessous

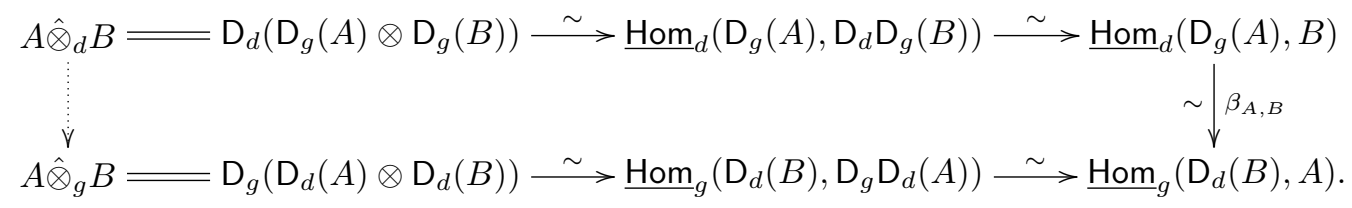




\section{La preuve du théorème 1.3}

On a le lemme technique suivant :

LEMME 3.1 - Le diagramme

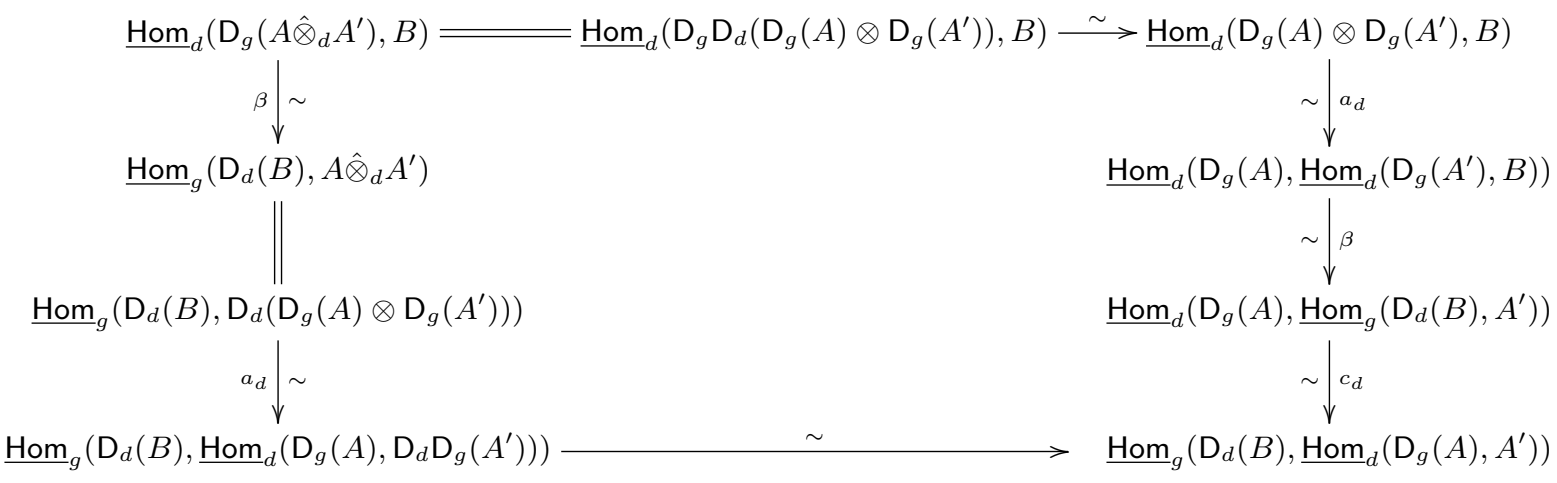

est commutatif pour tout $\left(A, A^{\prime}, B\right) \in \mathrm{Ob}(\mathcal{C})^{3}$.

Demonstration En apliquant le lemme 2.1 à $\mathrm{D}_{g}(A), \mathrm{D}_{g}\left(A^{\prime}\right), \mathrm{D}_{d}(B)$ et en évaluant en l'objet dualisant $R$, on obtient le diagramme commutatif

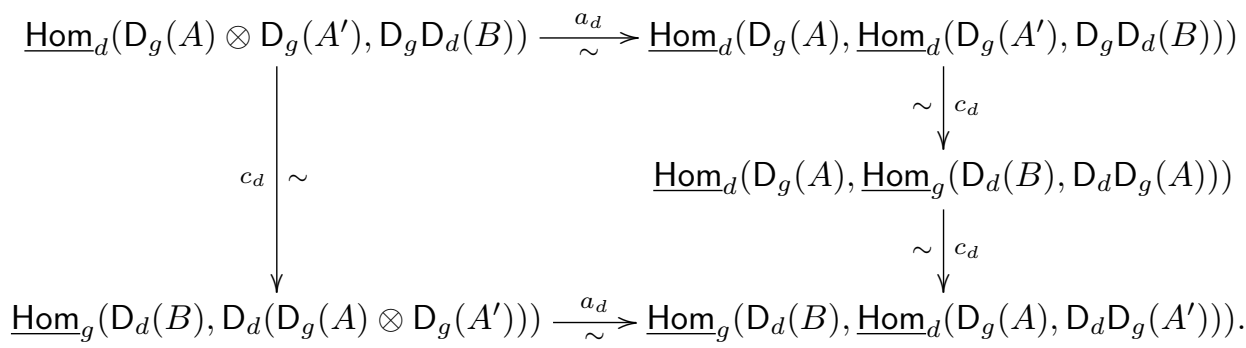

On peut alors former le diagramme commutatif

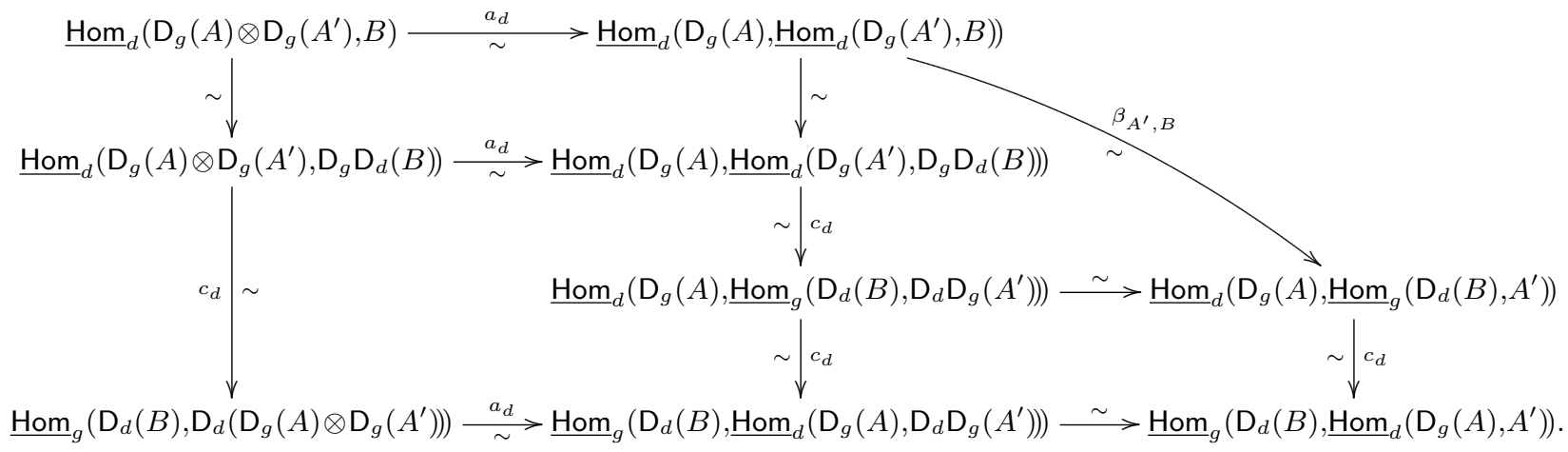

Or, les compositions de

$\underline{\operatorname{Hom}}_{d}\left(\mathrm{D}_{g}(A) \otimes \mathrm{D}_{g}\left(A^{\prime}\right), B\right) \stackrel{\sim}{\longrightarrow} \underline{\mathrm{Hom}}_{d}\left(\mathrm{D}_{g} \mathrm{D}_{d}\left(\mathrm{D}_{g}(A) \otimes \mathrm{D}_{g}\left(A^{\prime}\right)\right), B\right)$

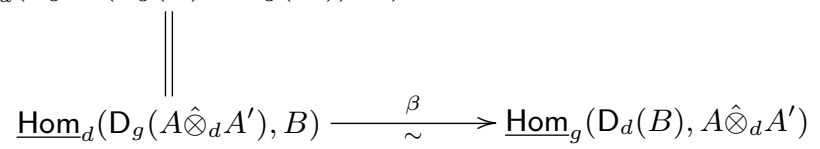

et $\quad \underline{\operatorname{Hom}}_{d}\left(\mathrm{D}_{g}(A) \otimes \mathrm{D}_{g}\left(A^{\prime}\right), B\right) \stackrel{\sim}{\longrightarrow} \underline{\operatorname{Hom}}_{d}\left(\mathrm{D}_{g}(A) \otimes \mathrm{D}_{g}\left(A^{\prime}\right), \mathrm{D}_{g} \mathrm{D}_{d}(B)\right)$

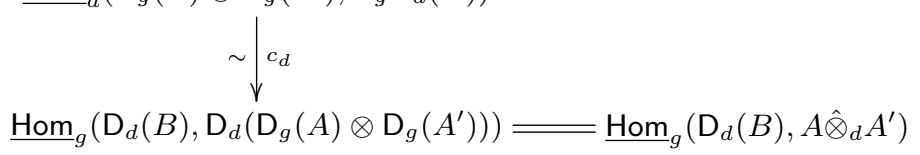

sont égales comme il découle immédiatement de la définition des isomorphismes $\beta$. Le lemme est démontré 
Dans le reste de la preuve, on utilisera librement les isomorphismes $A \hat{\otimes}_{g} B \simeq \underline{\operatorname{Hom}}_{g}\left(\mathrm{D}_{d}(B), A\right)$ et $A \hat{\otimes}_{d} B \simeq \underline{\operatorname{Hom}}_{d}\left(\mathrm{D}_{g}(A), B\right)$ égaux aux compositions des lignes du second diagramme de la définition 2.2. On note $\delta_{A, A^{\prime}, B}:\left(A \hat{\otimes}_{d} A^{\prime}\right) \hat{\otimes}_{g} B \stackrel{\sim}{\longrightarrow} A \hat{\otimes}_{d}\left(A^{\prime} \hat{\otimes}_{g} B\right)$ l'isomorphisme faisant commuter

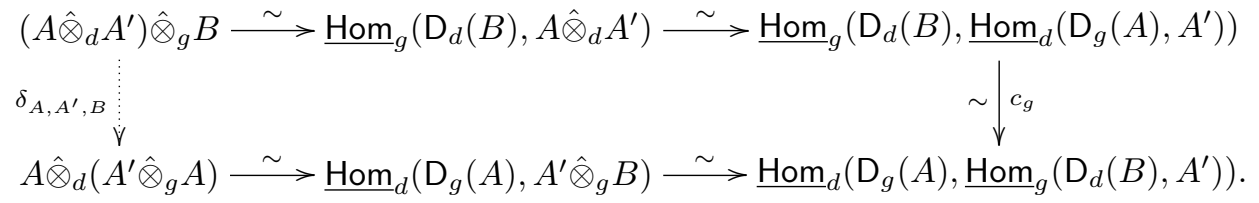

En composant certaines flèches dans diagramme commutatif de la proposition 3.1, on obtient le carré commutatif

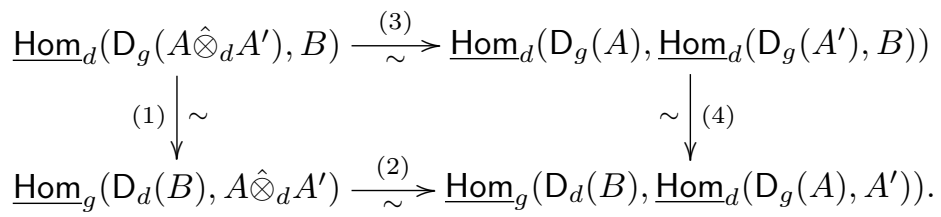

Les assertions ci-dessous sont faciles et leur vérification est laissée aux lecteurs.

1. Modulo les identifications

$$
\left(A \hat{\otimes}_{d} A^{\prime}\right) \hat{\otimes}_{d} B \simeq \underline{\operatorname{Hom}}_{d}\left(\mathrm{D}_{g}\left(A \hat{\otimes}_{d} A^{\prime}\right), B\right) \text { et }\left(A \hat{\otimes}_{d} A^{\prime}\right) \hat{\otimes}_{g} B \simeq \underline{\operatorname{Hom}}_{g}\left(\mathrm{D}_{d}(B), A \hat{\otimes}_{d} A^{\prime}\right),
$$

la flèche (1) correspond à $\gamma_{A \hat{\otimes}_{d} A^{\prime}, B}$.

2. Modulo l'identification $\left(A \hat{\otimes}_{d} A^{\prime}\right) \hat{\otimes}_{g} B \simeq \underline{\operatorname{Hom}}_{g}\left(\mathrm{D}_{d}(B), A \hat{\otimes}_{d} A^{\prime}\right)$ et l'identification composée de

$$
(\star) \quad A \hat{\otimes}_{d}\left(A^{\prime} \hat{\otimes}_{g} B\right) \simeq \underline{\operatorname{Hom}}_{d}\left(\mathrm{D}_{g}(A), A^{\prime} \hat{\otimes}_{g} B\right) \simeq \underline{\operatorname{Hom}}_{d}\left(\mathrm{D}_{g}(A), \underline{\operatorname{Hom}}_{g}\left(\mathrm{D}_{d}(B), A^{\prime}\right)\right.
$$

$$
\stackrel{c_{d}}{\sim} \underset{\operatorname{Hom}_{g}}{ }\left(\mathrm{D}_{d}(B), \underline{\operatorname{Hom}}_{d}\left(\mathrm{D}_{g}(A), A^{\prime}\right)\right),
$$

la flèche (2) correspond à $\delta_{A, A^{\prime}, B}$.

3. Modulo l'identification $\left(A \hat{\otimes}_{d} A^{\prime}\right) \hat{\otimes}_{d} B \simeq \underline{\operatorname{Hom}}_{d}\left(\mathrm{D}_{g}\left(A \hat{\otimes}_{d} A^{\prime}\right), B\right)$ et l'identification composée de

$$
\left(\star^{\prime}\right) \quad A \hat{\otimes}_{d}\left(A^{\prime} \hat{\otimes}_{d} B\right) \simeq \underline{\operatorname{Hom}}_{d}\left(\mathrm{D}_{g}(A), A^{\prime} \hat{\otimes}_{d} B\right) \simeq \underline{\operatorname{Hom}}_{d}\left(\mathrm{D}_{g}(A), \underline{\operatorname{Hom}}_{d}\left(\mathrm{D}_{g}\left(A^{\prime}\right), B\right)\right),
$$

la flèche (3) correspond au morphisme d'associativité de $\hat{\otimes}_{d}$.

4. Modulo les identifications composées de $(\star)$ et $\left(\star^{\prime}\right)$, la flèche $(4)$ correspond à id $\hat{\otimes}_{d} \gamma_{A^{\prime}, B}$. On a donc le résultat suivant :

Corollaire 3.2 - Pour tout $A, B$ et $C$ dans $\mathcal{C}$ le carré

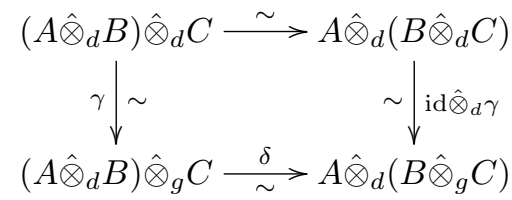

est commutatif.

En utilisant la $\otimes$-dualité, on obtient un deuxième carré commutatif. En effet, soit $\otimes^{\circ}$ la structure monoïdale opposée sur $\mathcal{C}$, i.e., celle donnée par $A \otimes^{\circ} B=B \otimes A$. On a alors :

$-{\widehat{\left(\otimes^{\circ}\right)_{g}}}=\left(\hat{\otimes}_{d}\right)^{\circ}$ et ${\widehat{\left(\otimes^{\circ}\right)_{d}}}=\left(\hat{\otimes}_{g}\right)^{\circ}$

- Les isomorphismes $\gamma_{A, B}$ et $\delta_{A, B, C}$ construit à partir de $\left(\mathcal{C}, \otimes^{\circ}\right)$ correspondent aux inverses de $\gamma_{B, A}$ et $\delta_{C, B, A}$ construit à partir de $(\mathcal{C}, \otimes)$.

Ainsi, en appliquant le corollaire 3.2 à $\left(\mathcal{C}, \otimes^{\circ}\right)$ on obtient le carré commutatif

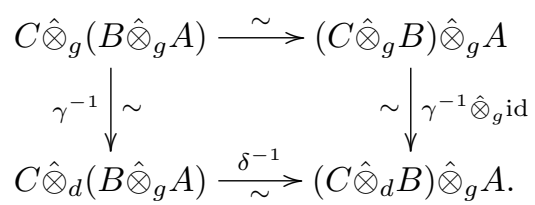


En inversant les flèches et en permutant les objets $A$ et $B$ on déduit le carré commutatif

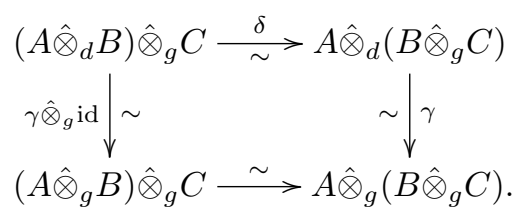

En recollant ce dernier carré avec celui du corollaire 3.2, nous obtenons le diagramme commutatif

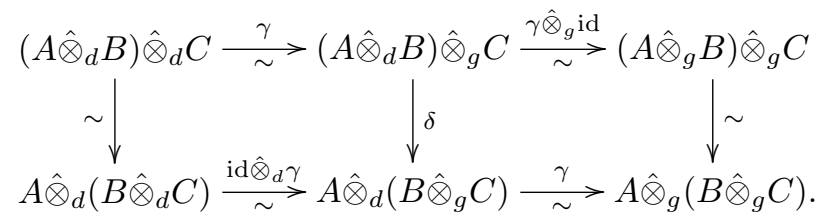

Or, le carré suivant

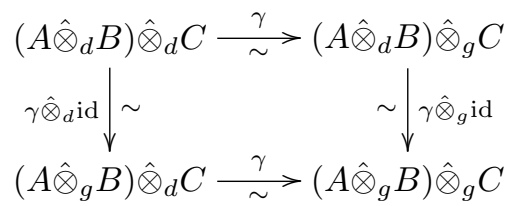

commute pour des raisons évidentes. On obtient en fin de compte la commutativité du diagramme

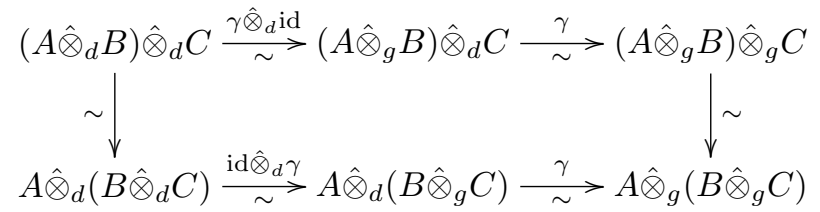

prouvant ainsi le théorème 1.3.

\section{Références}

[1] J. Ayoub. - Les six opérations de Grothendieck et le formalisme des cycles évanescents dans le monte motivique, I. Astérisque, volume 314, Société Mathématique de France, 2007.

[2] S. MacLane. - Natural associativity and commutativity. Rice University Studies 49, no. 4, p. 28-46, 1963.

Joseph Ayoub, Institut für Mathematik, Universität Zürich, Winterthurerstr. 190, CH-8057 Zürich, Switzerland E-mail : joseph.ayoub@math.uzh.ch 